\title{
Detección de personas en riesgo de padecer diabetes en farmacias comunitarias españolas
}

\author{
José A. Fornos-Pérez ${ }^{1,7,8}$, N. Floro Andrés-Rodríguez ${ }^{2,7,8}$, Blanca Lorenzo-Veiga ${ }^{3,7,8}$, Joaquina Huarte-Royo ${ }^{4,7}$, \\ Marta Vivar-Fernández ${ }^{5,7}$, J. Carlos Andrés-Iglesias ${ }^{3,7,8}$, Patricia García-Rodríguez ${ }^{6,7,8}$ \\ 1. Doctor en Farmacia. Farmacéutico comunitario en Cangas do Morrazo (Pontevedra). 2. Doctor en Farmacia. Farmacéutico comunitario en Vigo. \\ 3. Farmacéutico comunitario en Vigo. 4. Farmacéutica comunitaria en Pamplona. 5. Farmacéutica comunitaria en Barcelona. 6. Farmacéutico \\ comunitario en Cangas do Morrazo (Pontevedra). 7. Grupo de Diabetes de SEFAC. 8. Grupo Berbés de Investigación y Docencia.
}

\section{PALABRAS CLAVE}

Diabetes mellitus tipo 2, cribado, riesgo, Findrisc, farmacia comunitaria

\section{ABREVIATURAS}

ADA: American Diabetes Association. DE: desviación estándar. DM: diabetes mellitus. DM2: diabetes mellitus tipo 2.

GAA: glucosa alterada en ayunas.

GC: glucemia capilar. HbA1c: hemoglobina glucosilada.

IMC: índice de masa corporal.

NSC: National Screening Committee. SNC: sistema nervioso central.

TAG: tolerancia alterada a la glucosa.

\section{KEYWORDS}

Type 2 diabetes mellitus, screening, risk, Findrisc, community pharmacy

\section{RESUMEN}

Objetivo: Detectar personas con riesgo alto/muy alto de padecer diabetes y derivarlas al médico, evaluar en la muestra la prevalencia de los distintos factores de riesgo y realizar una intervención educativa mínima sobre éstos en todos los usuarios participantes.

Material y métodos: Estudio observacional transversal realizado en noviembre de 2014. Se incluyeron usuarios de la farmacia, mayores de 18 años, no diagnosticados de diabetes y que aceptaron realizar la encuesta. Muestreo no probabilístico.

Variable principal: puntuación obtenida en el cuestionario Findrisc. Otras: características demográficas, IMC, perímetro de cintura, glucemia capilar (si $\mathrm{F} \geq 15$ ), medicación, intervención, tiempo empleado.

Resultados: Participaron 90 farmacéuticos de las 17 comunidades autónomas. Realizaron 1.520 cuestionarios Findrisc. La puntuación media de la muestra fue de 10,9 $(\mathrm{DE}=5,1)$. El número de individuos con riesgo alto o muy alto fue de 370 (24,3\%) de los 1.520 encuestados. 207, el 55,9\% de aquellos y el $13,6 \%$ de la muestra total, tenían glucemia $\geq 110 \mathrm{mg} / \mathrm{dL}$ y se derivaron al médico. Existe relación directa entre el número de medicamentos utilizados y el riesgo de diabetes. El tiempo empleado en la intervención fue de 9,9 (DE=5,1) minutos.

Conclusiones: El alto porcentaje de participantes con riesgo alto/muy alto de padecer diabetes que son derivados al médico de familia para valorar su situación, avala la eficiencia de la farmacia en este tipo de cribados. La intervención educativa realizada con los participantes supone una llamada de atención sobre la importancia del estilo de vida saludable orientado a la prevención de las enfermedades metabólicas.

Detection of people at risk of suffering diabetes in Spanish community pharmacies

\section{ABSTRACT}

Objective: To detect people at high or very high risk of suffering diabetes and refer them to the physician; to evaluate the prevalence of the different risk factors in the study sample; and to target minimum educational intervention to such factors in all the participating pharmacy users.

Material and methods: A cross-sectional observational study was carried out in November 2014. Pharmacy users over 18 years of age, not diagnosed with diabetes and who agreed to participate in the survey were included in the study. Non-probabilistic sampling was made.

Primary endpoint: Findrisc questionnaire score. Others: demographic characteristics, body mass index, waist circumference, capillary blood glucose (if $\mathrm{F} \geq 15$ ), medication, intervention, time taken.

Results: Ninety pharmacists from the 17 Spanish Autonomous Communities participated. A total of 1520 Findrisc questionnaires were administered. The mean sample score was $10.9(\mathrm{SD}=5.1)$. Of the 1520 surveyed individuals, 370 were at high or very high risk (24.3\%). A total of 207 of these subjects (55.9\% and $13.6 \%$ of the global sample) presented blood glucose $\geq 110 \mathrm{mg} / \mathrm{dl}$ and were referred to the physician. There is a direct association between the number of medicines used and diabetes risk. The duration of the intervention was $9.9(\mathrm{SD}=5.1)$ minutes.

Conclusions: The large percentage of participants with a high or very high risk of suffering diabetes who are referred to the general practitioner for evaluation warrants the efficiency of pharmacies in screening initiatives of this kind. The described educational intervention underscores the importance of a healthy lifestyle in preventing metabolic diseases.

Un resumen de este trabajo se presentó como comunicación en formato póster en el XXVI Congreso Nacional de la Sociedad Española de Diabetes (SED) en Valencia, abril de 2015.

Recibido: $20 / 4 / 2015$

Aceptado: $30 / 5 / 2015$

Disponible online: 1-6-2015
Financiación: Acción financiada en el marco del Dia Mundial de la Diabetes en colaboración con la Federación de Diabéticos Españoles (FEDE). Conflicto de intereses: Los autores declaran no existir conflicto de intereses en relación con el contenido del artículo.

Cite este artículo como: Fornos-Pérez JA, Andrés-Rodríguez NF, Lorenzo-Veiga B, Huarte-Royo J, Vivar-Fernández M, Andrés-Iglesias JC, Garcia-Rodriguez P. Detección de personas en riesgo de padecer diabetes en farmacias comunitarias españolas. Farmacéuticos Comunitarios. 2015 Jun 01; 7(2):14-24 . doi:10.5672/FC.2173-9218.(2015Nol7).002.03

Autor para correspondencia: José A. Fornos Pérez (fornos@farmaciafornos.com) 


\section{Introducción}

En el estudio Di@bet.es de prevalencia de la diabetes mellitus (DM) en España [1] se encontró que casi el $30 \%$ de la población del estudio tenía algún tipo de alteración del metabolismo de los hidratos de carbono y que la prevalencia global de DM ajustada por edad y sexo era del 13,8\%, de los cuales algo menos de la mitad (6,0\%) tenía diabetes no conocida. Las tasas de prevalencia de la glucosa alterada en ayunas (GAA), tolerancia alterada a la glucosa (TAG) y combinado GAA-TAG ajustadas por edad y sexo fueron de 3,4\%, 9,2\% y 2,2\%, respectivamente. La prevalencia de la diabetes y las alteraciones de la regulación de la glucosa aumentan de manera significativa con la edad, siendo mayor en hombres que en mujeres [1].

Las personas con diabetes tipo 2 no diagnosticada presentan alto riesgo de padecer enfermedades cardiacas, dislipidemia, hipertensión y obesidad comparativamente con la población no diabética y por esta razón la detección precoz y el tratamiento inmediato reducen la gravedad de la enfermedad, así como las futuras complicaciones e ingresos hospitalarios [2-4]. Aunque existen contradicciones en la efectividad del cribado en cuanto a la reducción en la morbimortalidad en poblaciones con bajo riesgo de desarrollar DM2 [5-8], estudios realizados en varios países [8,9], en el nuestro [10] y organismos como el NSC (National Screening Committee) americano [11] o la American Diabetes Association (ADA) [12], recomiendan el cribado de riesgo de DM2.

En Europa, para detectar si una persona tiene riesgo o no de presentar diabetes en el futuro, se dispone de la escala Findrisc, que permite el cribado y el autocribado no invasivos [13,14]. Dicha escala ha sido traducida, adaptada y validada en varias poblaciones europeas [13]. Su validación para la población española fue realizada por Soriguer y cols. en 2012 [15,16].

Se trata de una herramienta fácil de utilizar, económica y rápida para el cribado en grandes grupos y estima la probabilidad de desarrollar diabetes tipo 2 en los próximos 10 años. Desde el punto de vista del farmacéutico comunitario, supone una oportunidad para promocionar estilos de vida saludables que modifiquen el estado de riesgo alto o retrasen la aparición de la enfermedad [17]. La sencillez de su cumplimentación ha favorecido su uso en campañas públicas de detección de la diabetes, así como en farmacias comunitarias [18].

Una puntuación en el cuestionario mayor o igual a 12 se relaciona con niveles de $\mathrm{HbA1c}$ iguales o mayores a 5,9\% [19], aunque el punto de corte más rentable para la predicción de un riesgo elevado de desarrollar diabetes ( $\geq 20 \%$ en 10 años) se obtiene a partir de los 15 puntos.

Las experiencias de cribados a sujetos no diagnosticados llevadas a cabo por farmacéuticos, en farmacias comunitarias [20-23], basadas en los factores de riesgo establecidos por la ADA [24] y con posterior determinación de glucemia basal en aquellos individuos con puntuación superior a 10 han resultado muy positivas.

Por ello, teniendo en cuenta la accesibilidad y proximidad a la población de las farmacias y los farmacéuticos comunitarios, el Grupo de Diabetes de la Sociedad Española de Farmacia Familiar y Comunitaria (SEFAC) junto con la Federación Española de Diabetes se plantearon un programa de detección de personas en riesgo de padecer DM utilizando el cuestionario Findrisc entre los usuarios que acuden a las farmacias.

\section{Objetivos}

\section{Objetivo general}

- Realizar un cribado de personas con riesgo de desarrollar diabetes utilizando el cuestionario Findrisc.

\section{Objetivos específicos}

- Detectar personas con riesgo alto a muy alto de padecer diabetes y derivarlas al médico.

- Evaluar en la muestra la prevalencia de los distintos factores de riesgo.

- Estudiar la relación del riesgo detectado mediante las puntuaciones del cuestionario con los factores de riesgo y características de los participantes.

- Realizar una intervención educativa mínima en todos los usuarios participantes sobre los factores de riesgo.

\section{Métodos \\ Diseño}

Estudio observacional transversal realizado en la semana del 10 al 17 de noviembre de 2014.
Ámbito

Se ofreció la participación como investigadores a todos los farmacéuticos comunitarios socios de SEFAC, dando preferencia a los miembros del Grupo de Diabetes de la Sociedad.

\section{Sujetos}

Criterios de inclusión: usuarios de la farmacia, mayores de 18 años no diagnosticados de diabetes y que aceptaron realizar la encuesta.

Criterios de exclusión: usuarios menores de 18 años y mayores de 18 años, que no estuvieran capacitados para cumplimentar el cuestionario o que no aceptasen hacerlo. Todos aquellos usuarios que hubieran sido diagnosticados de diabetes mellitus y/o tomasen medicamentos para la diabetes.

Muestreo no probabilístico. La incorporación al estudio se ofreció a las dos primeras personas que entraban en la farmacia por la mañana y por la tarde.

\section{Variables}

La variable principal es la puntuación obtenida en el cuestionario Findrisc. Se establecieron cinco subgrupos de pacientes de riesgo:

1. Riesgo bajo: menos de 7 puntos.

2. Riesgo ligeramente elevado: entre 7 y 11 puntos.

3. Riesgo moderado: entre 12 y 14 puntos.

4. Riesgo alto: entre 15 y 20 puntos.

5. Riesgo muy alto: más de 20 puntos.

Se realizaron las mediciones antropométricas necesarias para la cumplimentación del cuestionario:

- Índice de masa corporal (IMC): expresado en $\mathrm{kg} / \mathrm{m}^{2}$. Se utilizaron básculas electrónicas con tallímetro calibradas.

- Perímetro de cintura: expresado en $\mathrm{cm}$. Se midió con cinta métrica no extensible, según protocolo normalizado [25,26].

- Glucemia capilar: se realizó una determinación de glucemia capilar (GC) cuando la puntuación del cuestionario era $\geq 15$.

Se elaboró una hoja de registro $a d$ hoc (Figura 1) en la que se recogieron las respuestas al cuestionario Findrisc, las características demográficas de los sujetos, su medicación además de la antihipertensiva, la intervención realizada y el tiempo empleado. 

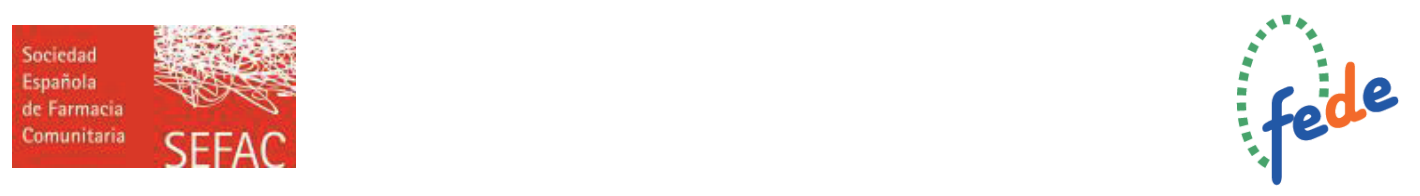

\section{¿CONOCE SU RIESGO DE PADECER DIABETES?}

\section{1. ¿Qué edad tiene?}

Menos de 45 años

45 - 54 años

55 - 64 años

Más de 64 años

\section{2. Índice de masa corporal (IMC)}

Menos de $25 \mathrm{Kg} / \mathrm{m}^{2}$

$25-30 \mathrm{Kg} / \mathrm{m}^{2}$

Mayor de $30 \mathrm{Kg} / \mathrm{m}^{2}$

\section{Perímetro de cintura}

$\frac{\text { Hombres }}{\text { Menos de } 94 \mathrm{~cm} \ldots \ldots . . . .} \frac{\text { Mujeres }}{\text { Menos }}$

Menos de $94 \mathrm{~cm} . . . \ldots . .$. Menos de $80 \mathrm{~cm} \quad 0$ puntos $\square$ $94-102 \mathrm{~cm}$.............. $80-88 \mathrm{~cm}$...... 3 puntos $\square$ Más de $102 \mathrm{~cm} \ldots \ldots .$. Más de $88 \mathrm{~cm} \ldots 4$ puntos $\square$

4. ¿Realiza diariamente al menos $\mathbf{3 0}$ minutos de actividad física, en el trabajo y/o en el tiempo libre?

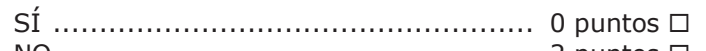

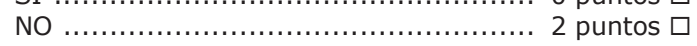

Escala de RIESGO TOTAL: puntos
5. ¿Con qué frecuencia toma verduras o frutas?

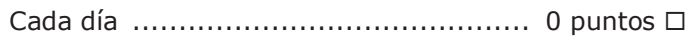

No cada día $\ldots \ldots \ldots \ldots \ldots \ldots \ldots \ldots \ldots \ldots, 1$ punto $\square$

6. ¿Toma medicamentos para la hipertensión de forma regular?

NO $\ldots \ldots \ldots \ldots \ldots \ldots \ldots \ldots \ldots \ldots \ldots \ldots \ldots \ldots, 0$ puntos $\square$

SI $\ldots \ldots \ldots \ldots \ldots \ldots \ldots \ldots \ldots \ldots \ldots \ldots \ldots \ldots \ldots \ldots \ldots \ldots \ldots, 2$ puntos $\square$

7. ¿Le han encontrado alguna vez valores de glucosa altos?

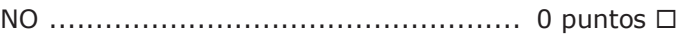

SI $\ldots \ldots \ldots \ldots \ldots \ldots \ldots \ldots \ldots \ldots \ldots \ldots \ldots \ldots \ldots \ldots \ldots \ldots \ldots, 5$ puntos $\square$

8. ¿Alguno de sus familiares, allegados u otros parientes han sido diagnosticados de diabetes?

NO ..................................... 0 puntos $\square$

SÍ: Abuelos, tíos, primos ...................... 3 puntos $\square$

SÍ: Padres, hermanos o hijos ............. 5 puntos $\square$

\begin{tabular}{|c|c|c|c|}
\hline & $\begin{array}{c}\text { Glucemia } \\
\text { basal }\end{array}$ & Hb1Ac & $\begin{array}{c}\text { TA } \\
\text { mmHg }\end{array}$ \\
\hline RIESGO MUY ALTO: Más de 20 puntos & & & \\
\hline RIESGO ALTO: Entre 15 y 20 puntos & & & \\
\hline RIESGO MODERADO: Entre 12 y 14 puntos & & & \\
\hline RIESGO LIGERAMENTE ELEVADO: Entre 8 y 11 puntos & & & \\
\hline RIESGO BAJO: Menos de 8 puntos & & & \\
\hline
\end{tabular}

\begin{tabular}{|c|c|c|c|c|}
\hline \multirow{2}{*}{\multicolumn{3}{|c|}{$\begin{array}{l}\text { Farmacia: } \\
\text { Paciente: }\end{array}$}} & \multicolumn{2}{|l|}{ Población: } \\
\hline & & & Código: & Tfno: \\
\hline Hombre $\square$ & \multicolumn{2}{|c|}{ Fumador: Sí $\square \quad$ No $\square$} & \multicolumn{2}{|c|}{ Evaluación/Intervención: } \\
\hline \multirow{4}{*}{\multicolumn{3}{|c|}{ Medicamento }} & & \\
\hline & & & $\begin{array}{l}\square \text { Educación sanitaria y } \\
\square \text { Educación sanitaria y } \\
\square \text { Derivación al médico y } \\
\square \text { Tiempo de la interven }\end{array}$ & $\begin{array}{l}5 \text { años } \\
\text { al año } \\
\text { guimiento } \\
\text { ר: minutos }\end{array}$ \\
\hline & & & Firma: & \\
\hline & & & Farmacéutico col. nº: & Fecha: \\
\hline \multicolumn{4}{|c|}{ Médico de familia (Información para el farmacéutico) } & Fecha: \\
\hline
\end{tabular}

(R) Grupo Berbés de Investigación y Docencia

Figura 1 Hoja de registro de datos, incluyendo el cuestionario Findrisc 


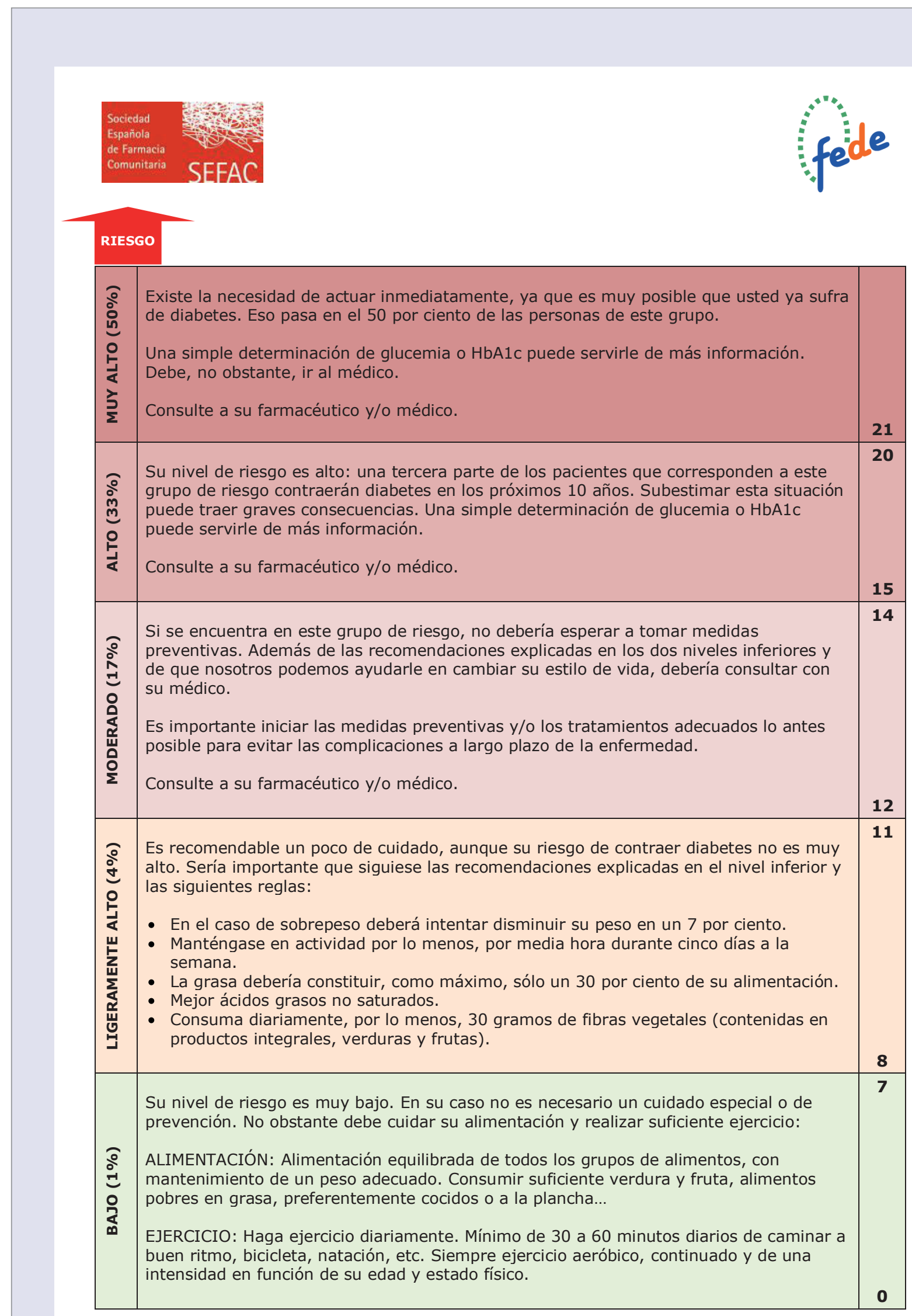

® Grupo Berbés de Investigación y Docencia

Figura 1 (Continuación) Hoja de registro de datos, incluyendo el cuestionario Findrisc 


\section{Procedimiento}

1. Captación de los participantes entre los días 10 y 17 de noviembre de 2014, ambos incluidos. Se informaba a los usuarios de la farmacia sobre el riesgo de padecer diabetes en el futuro y a los seleccionados se les ofrecía participar en el estudio. Se colocaron carteles informativos (Figura 2) y se llevó a cabo una captación activa.

2. Registro de los datos del participante, administración del cuestionario y cumplimentación, realizando las medidas antropométricas correspondientes (peso, talla y perímetro de cintura), incluidas en el cuestionario Findrisc, determinando el grado de riesgo de padecer DM.
3. Si la puntuación del cuestionario de Findrisc era $<15$ se le proporcionó educación sanitaria basada en hábitos higiénico-dietéticos saludables y se le recomendó la repetición del cuestionario al cabo de 5 años. A todos los usuarios participantes se les entregaron las fichas de recomendaciones de SEFAC sobre diabetes y estilos de vida saludables.

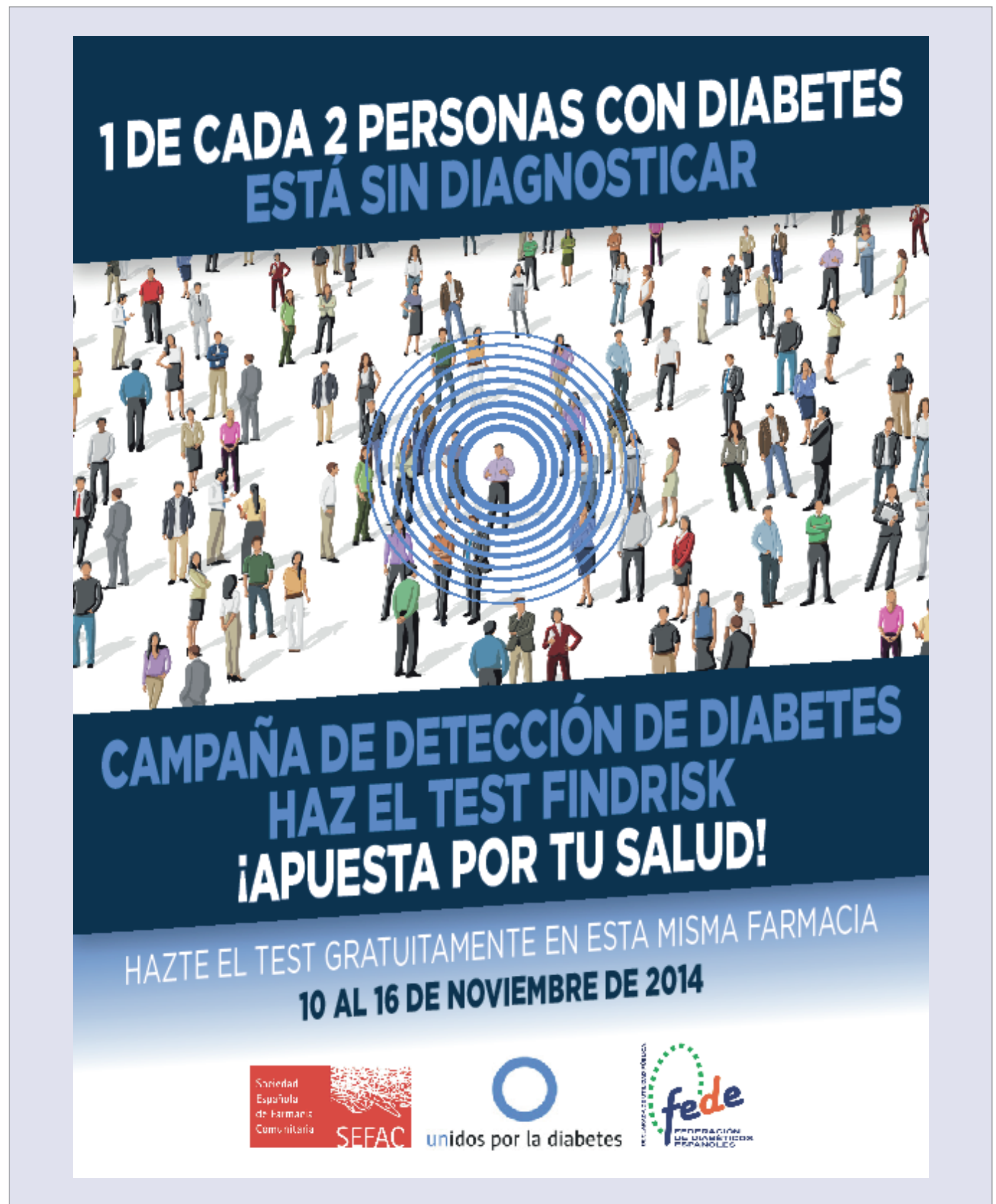

Figura 2 Cartel informativo 
4. Si la puntuación del cuestionario resultaba $\geq 15$, se le propuso realizar una determinación de la glucemia capilar. Los participantes con GC $\geq 110$ $\mathrm{mg} / \mathrm{dL}$ se derivaron al médico para su valoración. A los que obtuvieron un resultado de $\mathrm{GC}<110 \mathrm{mg} / \mathrm{dL}$ se les entregaron las fichas de SEFAC sobre diabetes y estilos de vida saludables y se les recomendaba realizar una nueva GC al cabo de un año.

5. Las hojas de registro se cumplimentaron por duplicado, entregándose una copia al participante y quedándose el farmacéutico con otra. Los datos registrados se volcaron a un formulario en la página web de SEFAC.

\section{Tratamiento estadístico}

Se calculó media y desviación estándar de la puntuación total del cuestionario Findrisc y las frecuencias relativas para cada una de las demás variables categóricas del cuestionario, para el conjunto de la muestra, por sexo y edad. Las diferencias entre grupos se calcularon mediante la prueba chi cuadrado, la t de Student y/o Wilcoxon. Se consideró significativa cuando el valor de $\mathrm{p}<0,05$.

Finalmente se realizó un análisis de regresión logística no condicional con las variables que resultaron significativas en el análisis univariante, para estimar la contribución independiente de cada una de ellas a la presencia de riesgo elevado de diabetes ( $\geq 15$ puntos), que se consideró como variable dependiente.

\section{Consideraciones éticas}

El tratamiento de los datos para el estudio se realizó de forma anónima y agregada, sin que fuera posible la identificación de los participantes, que podían abandonar el estudio en cualquier momento.

\section{Resultados}

Participaron en el estudio 90 farmacéuticos de las 17 comunidades autónomas. Realizaron 1.520 cuestionarios Findrisc, con una media de $16,9(\mathrm{DE}=6,1)$ cuestionarios por farmacia.

Las características de la población estudiada, distribuida en sexos y el valor total, incluyendo las preguntas correspondientes al cuestionario Findrisc, se presentan en la tabla 1 .
Tabla 1 Características de la muestra estudiada

\begin{tabular}{|c|c|c|c|c|}
\hline Variable & $\begin{array}{c}\text { Mujeres } \\
\text { n (\% grupo) }(\% \text { total })\end{array}$ & $\begin{array}{c}\text { Hombres } \\
\mathrm{n}(\% \text { grupo) }(\% \text { total) }\end{array}$ & $\begin{array}{c}\text { Total } \\
\text { n (\% total) }\end{array}$ & $\mathrm{p}$ valor \\
\hline \multicolumn{4}{|l|}{ Edad } & $p=0,2748$ \\
\hline$<45$ & $279(27,7)(18,4)$ & $138(27,0)(9,1)$ & $417(27,4)$ & \\
\hline $45-54$ & $245(24,3)(16,0)$ & $104(20,4)(6,8)$ & $349(23,0)$ & \\
\hline $55-64$ & $212(21,0)(14,0)$ & $121(23,7)(8,0)$ & $333(21,9)$ & \\
\hline$>64$ & $273(27,0)(18,0)$ & $148(29,0)(9,7)$ & $421(27,7)$ & \\
\hline Total & $1.009(100,0)(66,4)$ & $511(100,0)(33,6)$ & $1.520(100,0)$ & \\
\hline \multicolumn{4}{|l|}{ IMC } & $p<0,0001$ \\
\hline$<25$ & $350(34,7)(23,0)$ & $107(20,9)(7,0)$ & $457(30,1)$ & \\
\hline $25-30$ & $409(24,8)(26,9)$ & $258(50,5)(17,0)$ & $667(43,8)$ & \\
\hline$>30$ & $250(40,5)(16,5)$ & $146(28,6)(9,6)$ & $396(26,1)$ & \\
\hline Total & $1.009(100,0)(66,4)$ & $511(100,0)(33,6)$ & $1.520(100,0)$ & \\
\hline
\end{tabular}

Perímetro de cintura

\begin{tabular}{|c|c|c|c|c|}
\hline $\mathrm{M}:<80 \mathrm{H}:<94$ & $238(23,6)(15,7)$ & $124(24,3)(8,2)$ & $362(23,8)$ & \\
\hline $\begin{array}{l}M: 80-88 \quad H: \\
94-102\end{array}$ & $297(27,4)(19,5)$ & $198(38,8)(13,0)$ & $495(32,6)$ & \\
\hline $\mathrm{M}:>88 \quad \mathrm{H}:>102$ & $474(47,0)(31,2)$ & $189(37,0)(12,4)$ & $663(43,6)$ & \\
\hline Total & $1.009(100,0)(66,4)$ & $511(100,0)(33,6)$ & $1.520(100,0)$ & \\
\hline \multicolumn{4}{|l|}{ Ejercicio } & $p=0,2645$ \\
\hline Si & $592(58,7)(39,0)$ & $315(61,6)(20,7)$ & $907(59,7)$ & \\
\hline No & $417(41,3)(27,4)$ & $196(38,4)(12,9)$ & $613(40,3)$ & \\
\hline Total & $1.009(100,0)(66,4)$ & $511(100,0)(33,6)$ & $1.520(100,0)$ & \\
\hline
\end{tabular}

Consumo de vegetales

\begin{tabular}{|c|c|c|c|c|}
\hline Todos los días & $782(77,5)(51,5)$ & $361(70,7)(23,8)$ & $1.143(75,3)$ & \\
\hline No todos los días & $227(22,5)(14,9)$ & $150(29,4)(9,9)$ & $377(24,8)$ & \\
\hline Total & $1.009(100,0)(66,4)$ & $511(100,0)(33,6)$ & $1.520(100,0)$ & \\
\hline \multicolumn{4}{|c|}{ Medicamentos HTA } & $p=0,1240$ \\
\hline Si & $314(31,1)(20,7)$ & $179(35,0)(11,8)$ & $1.027(67,6)$ & \\
\hline No & $695(68,9)(45,7)$ & $332(65,0)(21,8)$ & $493(32,4)$ & \\
\hline Total & $1.009(100,0)(66,4)$ & $511(100,0)(33,6)$ & $1.520(100,0)$ & \\
\hline
\end{tabular}

Glucemia alta previa

\begin{tabular}{|l|c|c|c|}
\hline Si & $154(15,3)(10,1)$ & $93(18,2)(6,1)$ & $247(16,2)$ \\
\hline No & $855(84,7)(56,3)$ & $418(81,8)(27,5)$ & $1.273(83,8)$ \\
\hline Total & $1.009(100,0)(66,4)$ & $511(100,0)(33,6)$ & $1.520(100,0)$ \\
\hline
\end{tabular}

\begin{tabular}{|c|c|c|c|c|}
\hline \multicolumn{4}{|c|}{ Antecedentes familiares } & \multirow{5}{*}{$p=0,8431$} \\
\hline No & $496(49,2)(32,6)$ & $259(50,7)(17,0)$ & $755(49,7)$ & \\
\hline $2^{\circ}$ Grado & $176(17,4)(11,6)$ & $85(16,6)(5,6)$ & $261(17,1)$ & \\
\hline $1^{\circ} \mathrm{Grado}$ & $337(33,4)(22,2)$ & $167(32,7)(11,0)$ & $504(33,2)$ & \\
\hline Total & $1.009(100,0)(66,4)$ & $511(100,0)(33,6)$ & $1.520(100,0)$ & \\
\hline \multicolumn{4}{|l|}{ Fuma } & $p=0,0365$ \\
\hline Si & $209(20,7)(13,8)$ & $130(25,4)(8,6)$ & $339(22,3)$ & \\
\hline No & $800(79,3)(52,6)$ & $381(74,6)(25,0)$ & $1.181(77,7)$ & \\
\hline Total & $1.009(100,0)(66,4)$ & $511(100,0)(33,6)$ & $1.520(100,0)$ & \\
\hline
\end{tabular}


El número medio de medicamentos fue de $1,3(\mathrm{DE}=1,8)$ en las mujeres y $1,2(\mathrm{DE}=1,7)$ en hombres. La diferencia no resultó significativa ( $\mathrm{p}=0,2590)$. Los grupos de medicamentos más utilizados se muestran en la tabla 2. La relación entre los medicamentos más utilizados para otras enfermedades crónicas y el riesgo de padecer diabetes se presenta en la tabla 3. Existe relación directa entre el número de medicamentos utilizados y el riesgo de diabetes según la puntuación de Findrisc, Rho=0,2993 ( $\mathrm{p}<0,0001)$.

La puntuación media del cuestionario Findrisc fue de 10,8 $(\mathrm{DE}=5,1)$ en mujeres y de 11,1 ( $\mathrm{DE}=5,1)$ en hombres. La diferencia no resultó significativa ( $p=0,2164)$. La puntuación media de la muestra fue de 10,9 $(\mathrm{DE}=5,1)$. El número de individuos con riesgo alto o muy alto (puntuación en el cuestionario Findrisc $\geq 15$ ) fue de 370 $(24,3 \%)$ de los 1.520 encuestados.

El riesgo medio de la muestra total en el tramo etario de 18 a 45 años fue de 7,1 $(\mathrm{DE}=4,4)$ y en el tramo de más de 45 años de 12,2 (DE=4,7). El número de usuarios con riesgo alto o muy alto mayores de 45 años fue de $339(30,8 \%)$ frente a $21(5,0 \%)$ en los menores de 45 años.

Se determinó la glucemia capilar a 635 participantes, resultando unos valores medios en mujeres de 101,7 mg/dL $(\mathrm{DE}=23,2)$ y de $106,2 \mathrm{mg} / \mathrm{dL}(\mathrm{DE}=24,8)$ en los hombres, diferencia que resultó significativa $(\mathrm{p}<0,0173)$. De los 370 participantes con riesgo alto/muy alto, se encontraron 207, el 55,9\% de éstos y el $13,6 \%$ de la muestra total, con glucemia mayor o igual a $110 \mathrm{mg} / \mathrm{dL}$ y por lo tanto se derivaron al médico.

La estratificación del riesgo según las puntuaciones obtenidas y el sexo en la muestra total y en el tramo de más de 45 años se presenta en la tabla 4. En dicha tabla se muestra también el tipo de intervención realizado y el tiempo invertido por el farmacéutico en el conjunto de la actuación. A todos los usuarios participantes se les proporcionó educación sanitaria sobre la diabetes, orientada a la mejora de los hábitos alimenticios. Se les entregaron las fichas formativas de SEFAC (figura 3).

En el análisis multivariado se encontró que las variables no incluidas en el test de Findrisc, la utilización de antihipertensivos, hipolipemiantes y medicamentos para el sistema nervioso central (SNC), tienen valor pre-

Tabla 2 Utilización de los medicamentos más frecuentes

\begin{tabular}{|c|c|c|c|c|}
\hline Grupo terapéutico & $\begin{array}{c}\text { Mujer } \\
\text { n (\% grupo) (\% total) }\end{array}$ & $\begin{array}{c}\text { Hombre } \\
\text { n (\%grupo) (\% total) }\end{array}$ & Total & $\mathrm{p}$ valor \\
\hline \multicolumn{5}{|c|}{ Antihipertensivos } \\
\hline Si & $201(19,9)(13,2)$ & $147(28,8)(9,7)$ & $348(22,9)$ & \multirow{2}{*}{$p=0,0001$} \\
\hline \multirow[t]{2}{*}{ No } & $808(80,1)(53,2)$ & $364(71,2)(23,9)$ & $1.172(77,1)$ & \\
\hline & $1.009(100,0)(66,4)$ & $511(100,0)(33,6)$ & $1.520(100,0)$ & \\
\hline \multicolumn{5}{|l|}{ Hipolipemiantes } \\
\hline Si & $130(12,9)(8,6)$ & $79(15,5)(5,2)$ & $209(13,8)$ & \multirow{2}{*}{$p=0,1706$} \\
\hline \multirow[t]{2}{*}{ No } & $878(87,1)(57,8)$ & 433(84,5) $(28,4)$ & $1.311(86,2)$ & \\
\hline & $1.009(100,0)(66,4)$ & $511(100,0)(33,6)$ & $1.520(100,0)$ & \\
\hline \multicolumn{5}{|l|}{ Antiagregantes } \\
\hline Si & $27(2,7)(1,8)$ & $24(4,7)(1,6)$ & $51(3,4)$ & \multirow{2}{*}{$p=0,0388$} \\
\hline \multirow[t]{2}{*}{ No } & $982(97,3)(64,6)$ & $487(95,3)(32,0)$ & $1.469(96,6)$ & \\
\hline & $1.009(100,0)(66,4)$ & $511(100,0)(33,6)$ & $1.520(100,0)$ & \\
\hline \multicolumn{5}{|l|}{ SNC } \\
\hline Si & $135(13,4)(8,9)$ & $42(8,0)(2,7)$ & $175(11,6)$ & \multirow{2}{*}{$p=0,0021$} \\
\hline \multirow[t]{2}{*}{ No } & $874(86,6)(57,5)$ & $469(92,0)(30,9)$ & $1.343(88,4)$ & \\
\hline & $1.009(100,0)(66,4)$ & $511(100,0)(33,6)$ & $1.520(100,0)$ & \\
\hline
\end{tabular}

Tabla 3 Medicamentos y riesgo de padecer diabetes (puntuación Findrisc)

\begin{tabular}{|c|c|c|}
\hline $\begin{array}{c}\text { Grupo } \\
\text { terapéutico }\end{array}$ & Riesgo [m (DE)] & $p$ valor \\
\hline \multicolumn{3}{|c|}{ Antihipertensivos } \\
\hline Si & $13,6(4,5)$ & \multirow{2}{*}{$p<0,0001$} \\
\hline No & $9,7(4,7)$ & \\
\hline \multicolumn{3}{|c|}{ Hipolipemiantes } \\
\hline Si & $12,6(4,6)$ & \multirow{2}{*}{$p<0,0001$} \\
\hline No & $10,3(4,9)$ & \\
\hline \multicolumn{3}{|c|}{ Antiagregantes } \\
\hline Sí & $13,1(4,6)$ & \multirow{2}{*}{$p<0,001$} \\
\hline No & $10,5(4,9)$ & \\
\hline \multicolumn{3}{|l|}{ SNC } \\
\hline Sí & $12,0(4,7)$ & \multirow{2}{*}{$p=0,0001$} \\
\hline No & $10,4(5,0)$ & \\
\hline
\end{tabular}

dictivo de riesgo de diabetes (Aucroc) de $0,72,0,63$ y 0,59 respectivamente.

\section{Discusión \\ Limitaciones}

A pesar de que participaron farmacias de todas las autonomías españolas, la muestra no es representativa de la población nacional, lo que no era objetivo del estudio al tratarse de un pilotaje, por lo que los resultados son válidos tan solo para el conjunto de usuarios de la farmacia. El muestreo no probabilístico puede suponer una relativa limitación, que se compensa con la amplitud de la muestra.

En la administración del cuestionario Findrisc hay que tener en cuenta una posible sobrevaloración por el sujeto de sus hábitos en cuanto a ejercicio y contenido de vegetales y fruta en su dieta, por lo que el resultado de riesgo real podría ser ligeramente mayor que el obtenido.

Aunque en el procedimiento se estableció que la determinación de la glucemia capilar se haría solamente a los participantes con resultado del cuestionario $\mathrm{F} \geq 15$, la presión de los usuarios obligó a los farmacéuticos a realizarla a un número mayor de participantes (635). No obstante la derivación al médico, de acuerdo con la metodología acordada, se indicó solamente a los participantes con $\mathrm{F} \geq 15$ y glucemia capilar $\geq 110 \mathrm{mg} / \mathrm{dL}$.

Las características de la muestra son similares a las encontradas en otros estudios llevados a cabo en farmacias comunitarias [20-23]. Más del $66 \%$ son mujeres y cerca del $75 \%$ del total de encuestados tienen más de 45 años. El 70\% presenta sobrepeso u obesidad, cifras superiores a las estimadas para la población adulta en España [27] y más del 75\% tiene valores de perímetro de cintura superiores a la normalidad. Se encontró mayor porcentaje de obesidad entre las mujeres y entre los 
Tabla 4 Riesgo total, estratificación del riesgo, intervención y tiempo medio empleado en cada caso

\begin{tabular}{|c|c|c|c|c|}
\hline Variable & $\begin{array}{c}\text { Mujeres } \\
\text { n (\% grupo) }(\% \text { total })\end{array}$ & $\begin{array}{c}\text { Hombres } \\
\mathrm{n}(\% \text { grupo) (\% total) }\end{array}$ & $\begin{array}{c}\text { Total } \\
\text { n (\% total) }\end{array}$ & $\mathrm{p}$ valor \\
\hline \multicolumn{5}{|l|}{ Muestra total } \\
\hline Riesgo [m (DE)] & $10,8(5,1)$ & $11,1(5,1)$ & $10,9(5,1)$ & $p=0,2164$ \\
\hline Muy alto >20 & $49(4,9)(3,2)$ & $32(6,3)(2,1)$ & $81(5,3)$ & \multirow{5}{*}{$p=0,1188$} \\
\hline Alto $15-20$ & $200(19,8)(13,2)$ & $89(17,4)(5,9)$ & $289(19,0)$ & \\
\hline Moderado 12-14 & $190(18,8)(12,5)$ & $115(22,5)(7,6)$ & $305(20,1)$ & \\
\hline Ligero 7-11 & $287(28,4)(18,9)$ & $154(30,1)(10,1)$ & $441(29,0)$ & \\
\hline Bajo $<7$ & $283(28,1)(18,6)$ & $121(23,7)(8,0)$ & $404(26,6)$ & \\
\hline Total & $1.009(100,0)(66,4)$ & $511(100,0)(33,6)$ & $1.520(100,0)$ & \\
\hline \multicolumn{5}{|c|}{ Mayores de 45 años } \\
\hline Riesgo [m (DE)] & $12,1 \quad(4,7)$ & $12,4(4,8)$ & $12,2(4,7)$ & $p=0,2288$ \\
\hline Muy alto >20 & $46(6,3)(4,2)$ & $31(8,3)(2,8)$ & $77(7,0)$ & \multirow{5}{*}{$p=0,0707$} \\
\hline Alto $15-20$ & $182(24,9)(16,5)$ & $80(21,4)(7,2)$ & $262(23,8)$ & \\
\hline Moderado 12-14 & $152(20,8)(13,8)$ & $97(26,0)(8,8)$ & $249(22,6)$ & \\
\hline Ligero 7-11 & $222(30,4)(10,1)$ & $116(31,1)(10,5)$ & $338(30,6)$ & \\
\hline Bajo $<7$ & $128(17,5)(11,6)$ & $49(13,1)(4,4)$ & $177(16,1)$ & \\
\hline Total & $730(100,0)(66,2)$ & $373(10,0)(33,8)$ & $1.103(100,0)$ & \\
\hline \multicolumn{5}{|l|}{ Intervención } \\
\hline ES y $F$ a 5 años ${ }^{a}$ & $760(75,3)(50,0)$ & $390(76,3)(25,7)$ & $1.150(75,7)$ & \multirow{3}{*}{$p=0,0309$} \\
\hline ES y GC al añob & $127(12,6)(8,4)$ & $36(7,1)(2,3)$ & $163(10,7)$ & \\
\hline D al médico y $\varsigma^{c}$ & $122(12,1)(8,0)$ & $85(16,6)(5,6)$ & $207(13,6)$ & \\
\hline Total & $1.009(100,0)(66,4)$ & $511(100,0)(33,6)$ & $1.520(100,0)$ & \\
\hline Tiempo [m (DS)] & $9,7(5,1)$ & $10,2(5,1)$ & $9,9(5,1)$ & $p=0,0995$ \\
\hline
\end{tabular}

a Educación sanitaria y repetición del cuestionario Findrisc al cabo de 5 años.

- Educación sanitaria y repetición de la glucemia capilar al cabo de un año.

Derivación al médico y seguimiento del resultado de su evaluación.

hombres es mayor el porcentaje de sobrepeso y el perímetro de cintura.

Casi el 60\% afirma realizar al menos 30 minutos diarios de ejercicio, $y$ más del 75\% afirma consumir verduras y/o fruta todos los días. Como ya se señaló, son afirmaciones que deben aceptarse con cautela. Las mujeres afirman consumir verdura todos los días en mayor medida que los hombres. Los hombres afirman fumar más que las mujeres. No se encontraron diferencias significativas en las respuestas a los demás ítems del cuestionario.

Destaca la utilización de antihipertensivos en la muestra total y la mayor utilización de estos fármacos por los hombres (28,8\%). Asimismo, el uso de antiagregantes es mayor en los hombres y la de medicamentos para el SNC en las mujeres.
Se constató que los pacientes que utilizan antihipertensivos, hipolipemiantes, antiagregantes y medicamentos para patologías del sistema nervioso, presentaban mayor riesgo de padecer diabetes que los que no los utilizan. Se necesitaría un estudio con una muestra superior para confirmar si la utilización de esos medicamentos y/o las patologías para las que fueron prescritos, pudieran ser un factor de riesgo más a evaluar para la detección de diabéticos no diagnosticados.

La revisión de Waugh [11] en 2013, presenta estudios con distintas estrategias de cribado, la mayoría utilizando el cuestionario Findrisc. La Sociedad Española de Diabetes (SED) en su consenso [28] recomienda la utilización del cuestionario Findrisc en individuos de más de 40 años fijando como punto de corte $\mathrm{F} \geq 15$. Algunos estudios utilizan otras puntuaciones como punto de corte y así, por ejemplo, en el estudio Pizarra [15] se concluye que la mejor predicción del riesgo de incidencia de DM2 se produce en sujetos de más de 18 años con $\mathrm{F} \geq 9$ y glucemia basal $>100 \mathrm{mg} / \mathrm{dL}$.

En diversos países, con el mismo punto de corte, $\mathrm{F} \geq 15$, se encontraron desde un 9,6\% de personas con riesgo alto/muy alto de padecer diabetes, hasta un 45\% [29-32]. En el presente estudio fue del $24,3 \%$, pero hay que hacer constar que la edad de los participantes era de 18 años en adelante, cuando en la mayoría de los estudios, la edad es superior a 45 años y en alguno se limita a <65 años. En el estudio de Soriguer, de referencia por haber sido realizado en población española, la prevalencia del riesgo alto/muy alto fue del $14,1 \%$, en una muestra entre 18 y 65 años [15]. Se ha comprobado que el riesgo es mayor con la edad y que, desde el punto de vista de la eficiencia, el punto de corte serían los 45 años.

Por primera vez en un estudio de este tipo se ha valorado el tiempo que el farmacéutico comunitario invirtió en la administración del test de Findrisc y la intervención educativa realizada al participante, resultando en torno a los 10 minutos. En la cuantificación del coste de la actividad intervienen otros componentes que en este estudio no se han evaluado, por lo que será necesario profundizar en su análisis con el fin de conocer su coste real.

El propósito de este estudio fue llevar a cabo un pilotaje del cuestionario Findrisc en las farmacias comunitarias españolas, por lo que se priorizó la participación en él de farmacias de todas las comunidades autónomas españolas. El alto porcentaje de participantes con riesgo alto/muy alto de padecer diabetes que son derivados a su médico de familia con el fin de que este evalúe los resultados obtenidos, avala la eficiencia de la farmacia para la realización de este tipo de cribados ya que proporciona una oportunidad de realizar un diagnóstico temprano de una situación de alteración del metabolismo de la glucosa. La intervención educativa mínima realizada con todos los participantes supone una llamada de atención sobre la importancia de la consecución y mantenimiento de un estilo de vida saludable orientado a la prevención de las enfermedades metabólicas. 


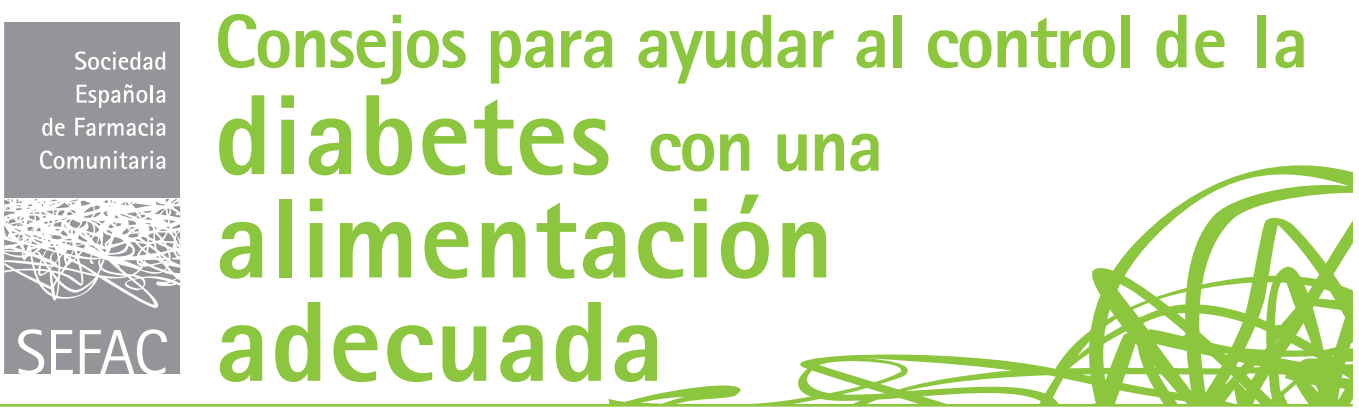

\section{Un pilar fundamental}

La alimentación equilibrada y el ejercicio adecuado a las necesidades de cada persona con diabetes, con el objetivo de conseguir un peso apropiado, son los pilares fundamentales para el tratamiento de esta patología, y se deben mantener aunque exista un tratamiento con medicamentos.

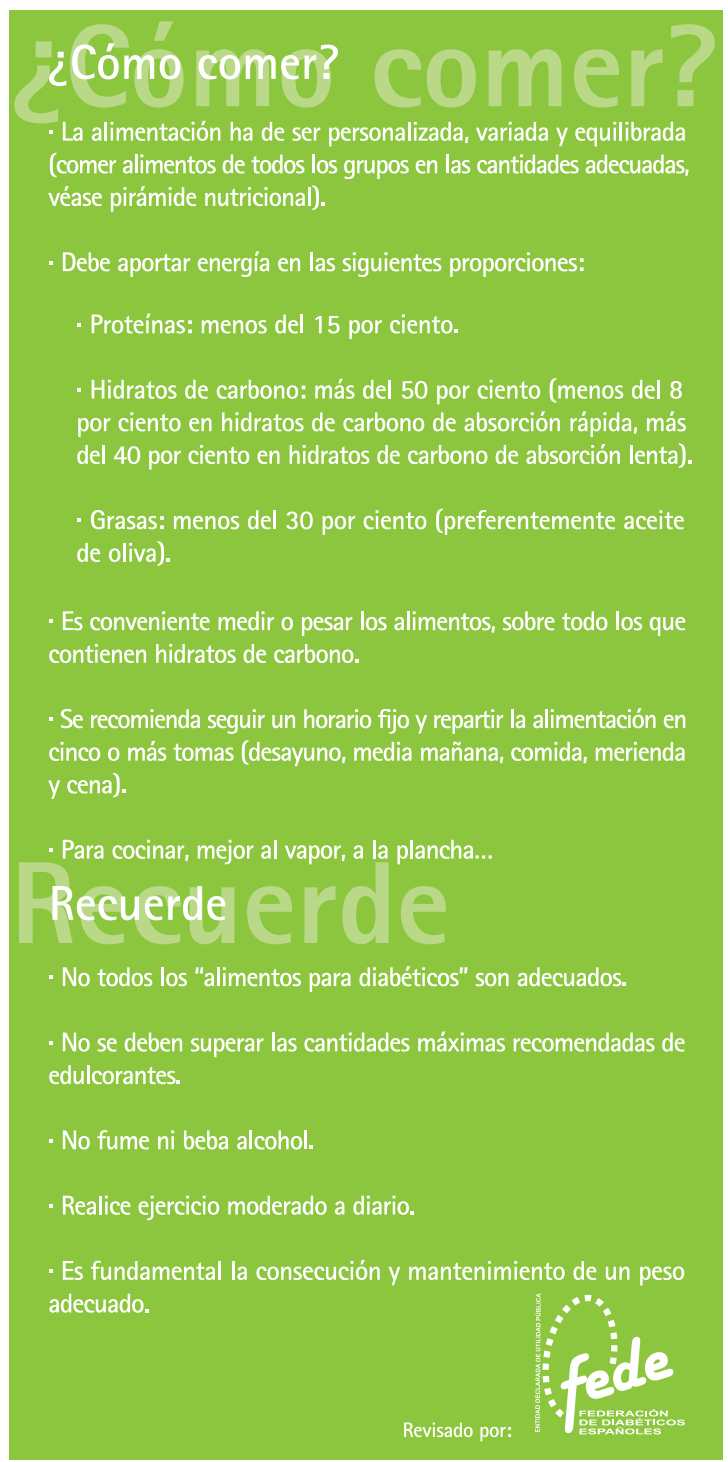

\section{¿Qué comer?}

- Utilizar alimentos ricos en fibra: verduras, legumbres, cereales integrales...

- Evitar / limitar los dulces.

- Reducir el consumo de grasas saturadas y colesterol (carnes rojas, visceras, embutidos, productos de bollería...).

- Es preferible el pescado a la carne y, en caso de dislipemias, limitar al máximo los mariscos.

- La leche y derivados (queso, yogur...), mejor desnatados.

- La sal no está prohibida, excepto en hipertensión y/o presencia de nefropatía diabética. En los demás casos, menos de tres gramos al día.

Pirámide de la alimentación: come sano y muévete

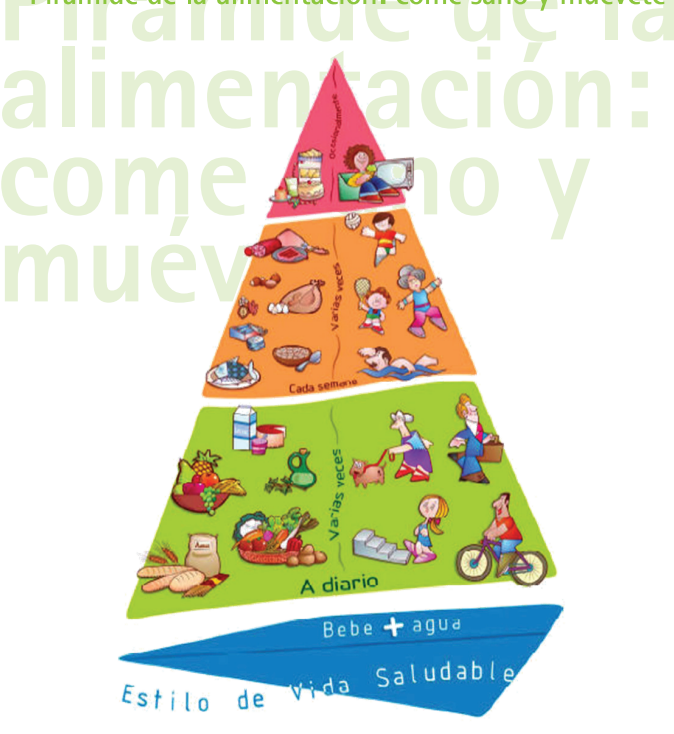

Fuente: Estrategia NAOS, Agencia Española de Seguridad Alimentaria y Nutrición.

Figura 3 Ficha formativa sobre DM de SEFAC 


\section{Referencias bibliográficas}

1. Soriguer F, Goday A, Bosch-Comas A, Bordiú E, Calle-Pascual A, Carmena $\mathrm{R}$, et al. Prevalence of diabetes mellitus and impaired glucose regulation in Spain: the Di@bet.es Study. Diabetología 2012;55(1):88-93.

2. Fornos Pérez JA, Fernández Cordeiro M, García Rodríguez P, González Añón D, Andrés Iglesias J, Andrés Rodríguez NF. Análisis de la utilización de medicamentos por los pacientes diabéticos en la provincia de Pontevedra. Av Diabetol. 2010;26:47-52. doi:10.1016/S1134-3230(10)61010-1

3. Lindenmeyer A, Hearnshaw H, Vermeire E, Van Royen P, Wens J, Biot Y. Interventions to improve adherence to medication in people with type 2 diabetes mellitus: a review of the literature on the role of pharmacists. J Clin Pharm Ther. 2006;31:409-419. doi:10.1111/j.1365-2710.2006.00759.x

4. Department of Noncommunicable Disease Management; World Health Organization. Screening for Type 2 Diabetes. Report of a World Health Organization and International Diabetes Federation meeting. Geneva (Switzerland): World Health Organization; 2003.

5. Simmons RK, Rahman M, Jakes RW, Yuyun MF, Niggebrugge AR, Hennings $\mathrm{SH}$, et al. Effect of population screening for type 2 diabetes on mortality: long-term follow-up of the Ely cohort. Diabetologia. 2011;54(2):312319. doi:10.1007/s00125-010-1949-8

6. Klein Woolthuis EP, de Grauw WJ, van Keeken SM, Akkermans RP, van de Lisdonk EH, Metsemakers JF, et al. Vascular outcomes in patients with screen-detected or clinically diagnosed type 2 diabetes: Diabscreen study follow-up. Ann Fam Med. 2013;11(1):20-27. doi:10.1370/ afm. 1460

7. Simmons RK, Echouffo-Tcheugui JB, Sharp SJ, Sargeant LA, Williams KM, Prevost AT, et al. Screening for type 2 diabetes and population mortality over 10 years (ADDITION-Cambridge): a cluster-randomized controlled trial. Lancet. 2012;380(9855):1741-1748. doi:10.1016/S0140-6736(12)61422-6

8. Park P, Simmons RK, Prevost AT, Griffin SJ. Screening for type 2 diabetes is feasible, acceptable, but associated with increased short-term anxiety: a randomized controlled trial in British general practice. BMC Public Health. 2008 Oct 7;8:350. doi:10.1186/14712458-8-350

9. Eborall HC, Griffin SJ, Prevost AT, Kinmonth AL, French DP, Sutton S Psychological impact of screening for type 2 diabetes: controlled trial and comparative study embedded in the ADDITION (Cambridge) randomized controlled trial. BMJ. 2007;335(7618):486. doi:10.1136/bmj. 39303.723449.55

10. Franch Nadal J, Artola Menéndez S, Díez Espino J, Mata Cases M. Evolución de los indicadores de calidad asistencial al diabético tipo 2 en atención primaria (1996-2007). Programa de mejora continua de calidad de la Red de Grupos de Estudio de la Diabetes en Atención Primaria de la Salud. Med Clin (Barc). 2010;135(13):600-607. doi:10.1016/j. medcli.2009.06.033

11. Waugh NR, Shyangdan D, Taylor-Phillips S, Suri G, Hall B. Screening for type 2 diabetes: a short report for the National Screening Committee. Health Technol Assess. 2013;17(35):1-90. doi:10.3310/hta17350

12. American Diabetes Association. Strategies for improving Care. Diabetes Care 2015;38(Suppl.1):S5-S7. doi:10.2337/dc15-S004

13. Lindstroem J, Tuomilehto J. The diabetes risk score: a practical tool to predict type 2 diabetes risk. Diabetes Care 2003;26:725-731. doi:10.2337| diacare.26.3.725

14. Saaristo T, Peltonen M, Keinänen-Kiukaanniemi S, Vanhala $M$, Saltevo J, Niskanen L, et al. FIN-D2D Study Group. Int J Circumpolar Health $2007 ; 66: 101-112$. doi:10.3402/ijch. v66i2.18239

15. Soriguer F, Valdés S, Tapia MJ, Esteva I, Ruiz de Adana MS, Almaraz MC, et al. Validación del FINDRISC (FINnish Diabetes Risk SCore) para la predicción del riesgo de diabetes tipo 2 en una población del sur de España. Estudio Pizarra. MedClin (Barc). 2012;138(9):371-376. doi:10.1016/j. medcli.2011.05.025

16. Soriguer F, Rojo-Martínez G, Almaraz MC, Esteva I, Ruiz de Adana MS, Morcillo S, et al. Incidence of type 2 diabetes in southern Spain (Pizarra Study). Eur J Clin Invest. 2008;38:126-133. doi:10.1111/j.13652362.2007.01910.x

17. Tomé Pérez Y, Justo Gonçalves MJ, Andrada Carretero B, Grajera Paredes ME, Cordón Arroyo A, Ferrer Aguareles JL. Cribado no invasivo de la diabetes tipo 2 en una muestra oportunista. Mérida: Servicio de Planificación y Coordinación Sanitaria. Junta de Extremadura; 2010

18. Fornos Pérez JA, Andrés Rodríguez NF, Andrés Iglesias JC, Acuña Ferradanes A, Costas González D, Mera Gallego R. Detección de pacientes con riesgo de desarrollar diabetes en farmacias comunitarias de Pontevedra. Farmacéuticos Comunitarios. 2013;5(3):141-146

19. Martin E, Ruf E, Landgraf R, Hauner $\mathrm{H}$, Weinauer F, Martin S. Findrisc questionnaire combined with $\mathrm{HbA} 1 \mathrm{c}$ testing as a potential screening strategy for undiagnosed diabetes in a healthy population. Horm Metab Res. 2011 0ct;43(11):782-787. doi:10.1055/s-0031-1286333

20. Guerra García MM, Fornos Pérez JA, Andrés Rodríguez NF. Detección de diabéticos no diagnosticados en las farmacias comunitarias de la provincia de Pontevedra. Pharm Care Esp. 2003;5(4):166-169.

21. Arias JL, Linares FM, Santamaria JM. Detección de posibles diabéticos no diagnosticados en una farmacia comunitaria. Ars Pharmaceutica. 2007;48(2):187-200.

22. Snella KA, Canales AE, Irons BK, Sleeper-Irons RB, Villarreal MC, Levi-Derrick VE, et al. Pharmacy-and community-based screenings for diabetes and cardiovascular conditions in high-risk individuals. J Am Pharm Assoc. 2006;46(3):370-377. doi:10.1331/154434506777069598

23. Hersberger KE, Botomino A, Mancini M, Bruppacher R. Sequential screening for diabetes - evaluation of a campaign in Swiss community pharmacies. Pharm WorldSci. 2006;28(3):171-179. doi:10.1007/s11096-006-9034-6

24. American Diabetes Association. Screening for Type 2 Diabetes. Diabetes Care. 2004;27(Suppl.1):S11-S14. doi:10.2337/diacare.27.2007.S11

25. De Koning L, Merchant AT, Pogue J, Anand SS. Waist circumference and waist-to-hip ratio as predictors of cardiovascular events: meta-regression analysis of prospective studies. Eur Heart J. 2007;28:850-856. doi:10.1093/eurheartj/ehm026

26. Balkau B, Deanfield JE, Després JP, Bassand JP, Fox KAA, Smith Jr $\mathrm{SC}$, et al. International Day for the Evaluation of Abdominal Obesity (IDEA): a study of waist circumference, cardiovascular disease, and diabetes mellitus in 168.000 primary care patients in 63 countries. Circulation 2007;116:19421951. doi:10.1161/CIRCULATIONAHA. 106.676379

27. Rodríguez E, López B, López AM, Ortega RM. Prevalencia de sobrepeso y obesidad en adultos españoles. Nutrición Hospitalaria. 2011;26(2):355363.

28. Mata Cases M, Artola S, Escalada J, Ezkurra Loyola P, Ferrer García JC, Fornos Pérez JA, Girbés J, Rica I. Consenso sobre la detección y el manejo de la prediabetes. Grupo de trabajo de consensos y guías clínicas de la Sociedad Española de Diabetes Farmacéuticos Comunitarios. 2014;6(4):26-39.

29. Hellgren MI, Petzold M, Björkelund C, Wedel H, Jansson PA, Lindblad 
U. Feasibility of the FINDRISC questionnaire to identify individuals with impaired glucose tolerance in Swedish primary care. A cross-sectional population-based study. Diabet Med. 2012;29(12):1501-1505. doi:10.1111/ j.1464-5491.2012.03664.x

30. Salinero-Fort MA, Carrillo-de Santa Pau E, Abánades-Herranz JC, Dujovne-Kohan I, Cárdenas-Valladolid J. Grupo MADIABETES. Riesgo basal de diabetes mellitus en atención pri- maria según cuestionario FINDRISC, factores asociados y evolución clínica tras 18 meses de seguimiento. Rev Clin Esp. 2010;210(9):448-453. doi:10.1016/j.rce.2010.03.008

31. Makrilakis K, Liatis S, Grammatikou S, Perrea D, Stathi C, Tsiligros P. Validation of the Finnish diabetes risk score questionnaire for screening for undiagnosed type 2 diabetes, dysglycaemia and the metabolic syndrome in Greece. Diabetes Metab.
2011;37(2):144-151. doi:10.1016/j.diabet.2010.09.006

32. Costa B, Barrio F, Piñol JL, Cabré JJ, Mundet X, Sagarra R, et al. Shifting from glucose diagnosis to the new HbA1c diagnosis reduces the capability of the Finnish Diabetes Risk Score (FINDRISC) to screen for glucose abnormalities within a real-life primary healthcare preventive strategy. BMC Medicine. 2013;11:45. doi:10.1186/1741-7015-11-45 